\title{
Avaliação do conhecimento sobre criptosporidiose em uma amostra de médicos de Porto Alegre, Rio Grande do Sul
}

\section{Evaluation of knowledge about cryptosporidiosis in a sample of physichians from Porto Alegre, Rio Grande do Sul, Brazil}

\author{
Adilia M.P. Wiebbelling' \\ Adelina Mezzari² \\ Letícia K. Schenato ${ }^{3}$ \\ Vinicius T. Richter ${ }^{3}$ \\ Eduardo Siaim Bohme ${ }^{4}$
}

\begin{abstract}
unitermos
resumo

Criptosporidiose

Introdução: A criptosporidiose, parasitose intestinal causada pelo protozoário Cryptosporidium parvum, foi descrita pela primeira vez em 1976. Desde então já houve relatos em diversos países, inclusive no Brasil.

Imunodeficiência Usualmente manifesta-se com episódio agudo de diarréia aquosa prolongada, dor abdominal, náuseas e

Exame parasitológico de fezes vômitos, afetando imunocomprometidos, crianças em creches e aqueles que entram em contato com água contaminada. O diagnóstico pode ser realizado com um exame parasitológico de fezes através de coloração específica, desde que seja solicitada a pesquisa do protozoário. Objetivo: Testar o grau de conhecimento sobre a doença em uma amostra de médicos de Porto Alegre (RS). Material e métodos: Contataram-se 92 médicos de um total de 411 vinculados a dois hospitais universitários de Porto Alegre, 173 da Irmandade Santa Casa de Misericórdia de Porto Alegre (ISCMPA) e 238 do Hospital São Lucas da Pontifícia Universidade Católica do Rio Grande do Sul (HSLPUC/RS), no período de julho de 1999 a agosto de 2000. A amostra abrangeu 91 especialistas: 41 da medicina interna, 11 da gastroenterologia, seis das doenças infecto-parasitárias, 18 da pediatria, oito da medicina comunitária e sete da ginecologia e obstetrícia. Os médicos foram selecionados randomicamente, e a amostra, estratificada de acordo com a quantidade de médicos por especialidade, visto que este número era significativamente diferente. Como instrumento utilizou-se um questionário (Anexo) com questões de escolha simples e múltipla, aplicado como entrevista por um dos autores (ESB), de acordo com normatizações estabelecidas. Os dados coletados foram analisados estatisticamente através do Microsoft Excel ${ }^{\circledR} 97$. Resultados: O grupo de risco mais lembrado foi o de imunocomprometidos (88/91). No entanto apenas $33 / 91$ consideraram grupo de risco crianças em creches. O sintoma mais referido foi diarréia aquosa prolongada (70/91), seguida por dor abdominal (59/91), náuseas e vômitos (19/91). Cinqüenta e três dos entrevistados disseram que solicitariam algum teste caso suspeitassem da doença. Entretanto 33 acham que um exame parasitológico de fezes comum pode diagnosticar a doença. Entre os 91 entrevistados, somente 37 relataram não haver tratamento para a doença, e 83 admitiram que precisam de mais informações sobre a mesma. Conclusão: A criptosporidiose é uma doença pouco conhecida pelos médicos. Com isso, há o risco de sua prevalência ser subestimada em nosso meio.
\end{abstract}

\section{abstract}

Background: Cryptosporidiosis is an intestinal parasitosis, caused by the Cryptosporidium parvum protozoa, was described, by the first time, in 1976. Since then, there already had several reports, including in Brazil. In general, it appears with a severe episode of watery diarrhea, prolonged abdomen pain, nausea and vomit, affecting immunocompromised, nursery children and people contacting contaminated water. The diagnosis can be done with a parasitological stool test with specific stain, since the protozoa search is required. Objective: The goal of this work was to test the knowledge degree on the disease in a Porto Alegre physician's sample. In two university hospitals of Porto Alegre, the Irmandade Santa Casa de Misericórdia (ISCMPA) and St'Lucas Hospital of Pontifícia Universidade Católica of Rio Grande do Sul (HSLPUC-RS), there are 411 physicians that work on those hospitals, 173 of ISCMPA and 238 HSLPUC-RS. From 411 those, was applied an interview in 92 physicians of different specialities, from July 1999 to August 2000. Ninety one specialist physicians were interviewed: 41 in internal medicine, 11 in gastroenterology, 6 in infections parasitic diseases, 18 in pediatrics, 8 in community medicine and

7 in gynecology and obstetrics. The physicians were randomic select and stratific sample with the number for specialities physicians, since the number was diferent significatly. As an instrument was used a questinare (annex) with single and doble questions, it was applied for author (ESB) followed defined rules. The die was cast in the Microsoft Exce ${ }^{\circledR} 97$ for statistics annalysis. Results: The most remembered risk group was the immunocompromised one (88/91). However, only 33/91 consider the children in nursey as a risk group. The most remembered symptom was the prolonged watery diarrhea (70/91), followed by abdominal pain (59/91) and nausea and vomiting (19/91). Fifty three interviewed said that they would request some test if the disease were suspected. However,

33/91 they believe that a common parasitological stool test can diagnose the disease. Among 91 of the interviewed, only 37 reported that there is not a treatment for the disease and 83 admitted that they need more information about the disease. It was verified that cryptosporidiosis is a disease not well-known by the physicians, and, togheter there is the risk of its prevalence being underestimated in our environment.

\section{key words}

Cryptosporidiosis

Immunodeficiency

Parasitological stool test
1. Professora de Parasitologia da Fundação Faculdade Federal de Ciências Médicas de Porto Alegre (FFFCMPA); mestranda em Saude Coletiva na Universidade Luterana do Brasil.

2. Professora de Parasitologia da FFFCMPA e de Micologia na Faculdade de Farmácia da UFRCS. doutoranda em Ciências Veterinárias na UFRCS. 3. Doutorando do curso de Medicina da FFFCMPA.

4. Acadêmico e monitor da disciplina de Parasitologia da FFFCMPA.

Trabalho realizado na disciplina de Parasitologia do Departamento de Microbiologia e Parasitologia da FFFCMPA. 


\section{Introdução}

A criptosporidiose intestinal, uma infecção parasitária causada pelo Cryptosporidium parvum, foi descrita pela primeira vez em 1976 (1). Desde então já foi relatada em mais de 60 países (2), inclusive no Brasil.

A doença caracteriza-se por episódios de diarréia prolongada ou persistente, principalmente se comparada à de pacientes com diarréia aguda (16), associada a dor abdominal, náuseas, vômitos e, mais raramente, febre, mal-estar e anorexia. As fezes têm característica de diarréia aquosa sem sangue e/ou pus. Sua ocorrência está vinculada principalmente a pacientes imunodeprimidos, especialmente àqueles infectados com o vírus da imunodeficiência humana adquirida (HIV). Nestes pacientes, a doença pode ser potencialmente fatal (3).

Alguns surtos de criptosporidiose têm sido descritos (4-6). Nestes, a doença foi associada à exposição e ao consumo de água contaminada ou leite. Pode ser associada também à exposição de auxiliares de enfermagem a pacientes contaminados. Entretanto existe pouca ou nenhuma informação sobre sua incidência e seus fatores de risco $(6,7)$.

Muitos fatores podem contribuir para a falta de conhecimento sobre a sua epidemiologia. Entre eles destaca-se a ausência da prática de notificação da doença por parte dos médicos e dos laboratórios. Além disso, as técnicas diagnósticas utilizadas pela maioria dos laboratórios não permitem a identificação do parasita em um exame parasitológico de fezes rotineiro. Muitos testes foram desenvolvidos para identificar os oocistos do parasita, entretanto a realização destes só é feita mediante requisição do médico assistente.

\section{Material e método}

Para o estudo contataram-se 92 médicos, num total de 411 vinculados a dois hospitais universitários da cidade de Porto Alegre (RS), 173 da Irmandade Santa Casa de Misericórdia de Porto Alegre (ISCMPA) e 238 do Hospital São Lucas da Pontifícia Universidade Católica do Rio Grande do Sul (HSLPUC/RS). A amostra abrangeu 91 especialistas divididos nas seguintes áreas: medicina interna (41); gastroenterologia (11); doenças infecto-parasitárias (seis); pediatria (18); medicina comunitária (oito); e ginecologia e obstetrícia (sete). Os médicos foram selecionados randomicamente pelo quadro de funcionários das instituições. A amostra foi estratificada em especialidades, pois o número de médicos em cada especialidade foi significativamente diferente.
Como método de coleta utilizamos um questionário (Anexo) composto de questões de escolha múltipla ou simples. Este instrumento foi aplicado, como entrevista, somente por um dos autores (ESB), através de normatizações previamente estabelecidas. O questionário incluiu questões sobre sintomas associados, grupos de risco, conhecimentos sobre testes laboratoriais e tratamento da doença. Os entrevistados estimaram, ainda, a proporção de seus pacientes que é infectada pelo vírus do HIV, por serem estes pacientes os mais atingidos pela doença, que pode ser fatal (3).

Em seguida, os dados coletados foram enviados a um banco de dados informatizado (Microsoft Excel ${ }^{\circledR}$ 97), com análise estatística, sendo estes apresentados em tabelas de distribuição e contingência e gráficos.

\section{Resultados}

Dos 92 médicos abordados, 91 responderam ao questionário: 41 internistas, 11 gastroenterologistas, seis infectologistas, 18 pediatras, oito médicos comunitários e sete ginecologistas obstetras.

A média de tempo decorrido desde a graduação até a entrevista é baixa, se considerarmos que 77 dos 91 entrevistados eram médicos residentes nas especialidades. Quase todos os entrevistados concluíram seu curso em universidades públicas (66/91).

A maioria identificou corretamente diarréia aquosa prolongada (DAP) como principal sintoma da doença. Entre as especialidades, os médicos comunitários foram os que menos associaram DAP à doença. Entretanto poucos associaram dor abdominal e náuseas/vômitos à parasitose. Quase um quarto dos pediatras desconhecia qualquer sintoma de criptosporidiose.

Com relação aos grupos de risco, quase a totalidade dos entrevistados (85/91) identificou pacientes imunodeprimidos como de alto risco para adquirir a doença. Outros grupos, como viajantes, pessoas em contato com animais e crianças em creches, foram pouco relatados como de risco. Ainda, $63 \%$ dos médicos comunitários e $45 \%$ dos pediatras não identificaram crianças em creches como grupo de risco.

Como a criptosporidiose apresenta-se intimamente ligada à Aids, estimou-se seu percentual para compará-lo às informações que estes médicos têm sobre a parasitose. A especialidade que apresentou maior percentual de aidéticos, como esperado, foi a de doenças infecciosas e parasitárias, com uma média de aproximadamente $60 \%$ de seus pacientes. Mas somente $50 \%$ dos especialistas desta 
área salientaram com exatidão os três sintomas habituais da doença.

Muitos $(60,2 \%)$ acreditam que um exame parasitológico de fezes de rotina inclui pesquisa de oocistos do Cryptosporidium parvum. Entre as especialidades, $83 \%$ dos infectologistas solicitariam algum exame caso suspeitassem da doença. Entretanto somente $21,9 \%$ dos internistas, $37,5 \%$ dos gastroenterologistas, $33,3 \%$ dos pediatras, $37,5 \%$ dos médicos comunitários e $42,8 \%$ dos ginecologistas obstetras solicitariam algum teste específico se suspeitassem da doença. As principais razões para não solicitarem testes laboratoriais específicos foram: baixa ocorrência da doença $(23,8 \%)$, falta de familiaridade com a doença $(14,7 \%)$, falta de conhecimento dos testes $(12,5 \%)$ e custo elevado dos mesmos $(4,5 \%)$.

A maioria dos entrevistados $(94,3 \%)$ acha que precisa de mais informações sobre o tema. Por especialidade, $97,5 \%$ dos internistas, $72,7 \%$ dos gastroenterologistas, $33,3 \%$ dos infectologistas, $100 \%$ dos pediatras e médicos comunitários e $87,5 \%$ dos ginecologistas obstetras acham que necessitam conhecer melhor a doença.

\section{Discussão}

Os resultados deste estudo mostram que a maioria dos médicos entrevistados, atuantes em hospitais universitários de Porto Alegre, sabe que criptosporidiose intestinal manifesta-se com diarréia aquosa prolongada, especialmente em pacientes imunodeprimidos. No entanto não reconhecem outros sintomas da doença, como dor abdominal e náuseas/ vômitos, nem outros grupos de risco, tais como crianças em creches e pessoas em contato com animais. A diarréia aquosa prolongada é aceita como sintoma da doença por $62,5 \%$ dos médicos comunitários e $38,8 \%$ dos pediatras, e também $62,5 \%$ e, principalmente, $44,4 \%$ destes especialistas, respectivamente, não reconhecem crianças em creches como grupo em risco, apesar de, atualmente, as crianças de diferentes classes socioeconômicas permanecerem nestes estabelecimentos grande parte de seu dia. Assim, a doença pode estar sendo subestimada, especialmente em crianças.

Mesmo quando há suspeita diagnóstica correta da doença podem ocorrer falhas no processo. Muitas vezes isso acontece pelo desconhecimento dos testes laboratoriais relativos à doença. A maioria dos entrevistados $(60,2 \%)$ acha que os laboratórios são capazes de detectar o parasita em um exame parasitológico de fezes de rotina, sem requisição médica para a sua procura específica. Na verdade, nenhum laboratório realiza a procura do parasita se esta não for re- quisitada pelo médico assistente. A identificação laboratorial é importante, não somente para que o diagnóstico seja estabelecido, mas também para que dados epidemiológicos e programas de saúde pública possam ser ajustados, principalmente se levarmos em conta que o parasito é considerado um problema de saúde pública não só nos países subdesenvolvidos, como nos desenvolvidos também (17).

A razão mais freqüente apontada para a não-realização de testes laboratoriais, como o diagnóstico de criptosporidiose intestinal, foi a baixa ocorrência da doença. A maioria dos estudos de prevalência é limitada a populações selecionadas, como pacientes imunodeprimidos, crianças em creches, crianças hospitalizadas ou agricultores $(6,8-10,14)$. Entretanto a prevalência real da doença na população geral é desconhecida. Alguns trabalhos $(11,12)$ têm mostrado prevalência de $1 \%$ em amostras aleatórias de fezes na população geral, e o mesmo índice aparece em estudo só com crianças (15). Um estudo realizado em Connecticut, Estados Unidos, mostrou altos valores de positividade nas amostras (13), ou seja, 2,8\%, quando o exame específico foi solicitado. Entretanto todas as amostras suspeitas de criptosporidiose foram examinadas, independente de solicitação médica, e foram positivas em $5,8 \%$ dos casos. Conclui-se que os pacientes com alto risco de serem contaminados com a doença não estão sendo bem selecionados para a realização de testes laboratoriais. Isto pode ocorrer pelo fato de os médicos desconhecerem a necessidade de requisição do exame específico para o parasita.

\section{Conclusão}

É iminente a necessidade de melhora do conhecimento médico sobre criptosporidiose em nosso meio. Médicos de todas as especialidades, mas sobretudo aqueles ligados a populações de risco (médicos comunitários e pediatras), têm poucas informações sobre a apresentação e os fatores de risco da doença. Em quase todas as especialidades é pequeno o conhecimento sobre a necessidade de solicitar a pesquisa específica do parasita no exame parasitológico de fezes. Assim corremos o risco de subestimar a presença da doença entre nós e, com isso, o impacto que ela exerce sobre a saúde pública, principalmente se levarmos em consideração, como citado anteriormente, que o problema ocorre não somente em países subdesenvolvidos, mas nos desenvolvidos também (17).

Indubitável é a preocupação dos médicos entrevistados com a doença, pois a maioria se mostrou interessada em receber maiores informações sobre a mesma. 


\section{Referências}

I. Current, W.L. \& Garcia, L.S. Cryptosporidiosis. Clin. Microbiol. Rev., 4: 305-95, 1991.

2. Holley, H.P. \& Dover, C. Cryptosporidium: a common cause of parasitic diarrhea in otherwise healthy individuals. J. Infect. Dis., 153: 365-7, 1986.

3. Peterson, C. Cryptosporidiosis in patients infected with the immunodeficiency virus. Clin. Infect. Dis., 15: 903-7, 1992.

4. McAnulty, J.M.; Fleming, D.W. \& Gonzalez, A.H. A communitywide outbreak of cryptosporidiosis associated with swimming at a wave pool. Jama, 272: I597-600, 1994.

5. Mackenzie, W.R. et al. A massive outbreak in Milwaukee of Cryptosporidium infection transmitted throught the public water supply. NEJM, 33 I: 161-7, 1994.

6. Alpert, G. et al. Cryptosporidiosis in a day care center. NEJM, 311:860-I, 1984.

7. Navin, T.R. \& Juranek, D.D. Cryptosporidiosis: clinical, epidemiological and parasitologic review. Rev. Infect. Dis., 6: 3।3-27, 1984.

8. Crawford, F.G.;Vermund, S.H. \& Deckelbaum, R.J. Asymptomatic cryptosporidiosis in a New York day care center. Ped. Infect. Dis. J., 1 1: 806-7, 1988.

9. Ravn, P. et al. Nosocomial outbreak of cryptosporidiosis in Aids patients. BMJ, 302: 277-80, 1991.
I0.Thomson, M.A.; Benson, J.W.T. \& Wright, P.A.Two-year study of Cryptosporidium infection. Arch. Dis. Child., 62: 559-63, 1987.

I I. Skeels, M.R. et al. Cryptosporidium infection in Oregon public health clinic patients, 1985-88: the value of statewide laboratory surveillance. Am.J. Public. Health, 80: 305-8, 1990.

12. Hamoudi, A.C. et al. Do regional variances in prevalence of cryptosporidiosis occur?The central Ohio experience. Am. J. Public. Health, 78: 273-5, 1988.

13. Roberts, C.L. et al. Factors influencing Cryptosporidium testing in Connecticut. J. Clin. Microbiol., 34: 2292-3, 1996.

14. Franco, R.M.B. \& Cordeiro, N.S. Giardiose e criptosporidiose em creches no município de Campinas, SP. Rev. Soc. Bras. Med.Trop., 29(6): 585-91, 1996.

15. Oshiro, E.T. et al. Prevalência de Cryptosporidium parvum em crianças abaixo de 5 anos, residentes na zona urbana de Campo Grande, MS, Brasil, 1996. Rev. Soc. Bras. Med. Trop., 33(3): 277-80, 2000.

16. Newman, R.D. et al. Longitudinal study of Cryptosporidium parvum infection in children in northeastern Brazil. J. Inf. Dis., 180: 167-75, 1999.

17. Newman, R.D. et al. Household epidemiology of Cryptosporidium parvum infection in an urban comunity in northeast Brazil. Ann. Intern. Med., I 20: 500-5, 1994. 


\section{Anexo - Questionário aplicado aos médicos}

\section{Fundação Faculdade Federal de Ciências Médicas de Porto Alegre}

\section{Disciplina de Parasitologia}

\section{Questionário sobre criptosporidiose intestinal}

1. Especialidade médica:
( ) Medicina interna
( ) DIP
( ) Gastroenterologia
( ) Pediatria
( ) Medicina comunitária

2. Ano de graduação: 19

3. Local de graduação:

( ) Faculdade pública da região metropolitana de Porto Alegre

( ) Faculdade privada da região metropolitana de Porto Alegre

4. Selecione os três (3) sintomas mais importantes que o levariam a pensar num quadro clínico típico de criptosporidiose intestinal:
( ) Diarréia com sangue
( ) Diarréia aquosa prolongada
( ) Diarréia com pus
( ) Dor abdominal
( ) Tenesmo
( ) Prurido anal
( ) Febre
( ) Náuseas e vômitos
( ) Não sabe
( ) Emagrecimento
( ) Astenia

5. Selecione os grupos de pessoas que considera com risco aumentado para infecção intestinal por Cryptosporidium (escolha múltipla):
( ) Pacientes imunocomprometidos
( ) Ordenhadores
( ) Crianças que freqüentam creches
( ) Funcionários de limpeza pública
( ) Viajantes
( ) Pessoas que mantêm contato com animais
( ) Auxiliares de enfermagem $(\%)$.

6. Estime a proporção de seus pacientes que são portadores do vírus da Sida:

7. Você costuma solicitar algum teste diagnóstico para criptosporidiose quando suspeita da doença?

( ) Sim. Qual(is)?

( ) Não

8. Você acha que é possível diagnosticar criptosporidiose com um exame parasitológico de fezes de rotina para achado de oocistos?
( ) Sim
( ) Não

9. Se você respondeu não à pergunta 7, selecione a(s) razão(ões) para não fazê-lo:

( ) Baixa ocorrência de criptosporidiose entre seus pacientes

( ) Falta de familiaridade com a doença

( ) Falta de conhecimento sobre testes diagnósticos para a doença

( ) Custo elevado dos testes

( ) Falta de colaboração dos pacientes para realizar os testes

( ) Outra:

10. Selecione a(s) melhor(es) opção(ões) para o tratamento da criptosporidiose:
( ) Mebendazol
( ) Albendazol
( ) Levamisol
( ) Metronidazol
( ) Praziquantel
( ) Sulfametoxazol-Trimetropin
( ) Furazolidona
( ) Não há tratamento efetivo até o momento

11. Você acha que precisa de informações adicionais sobre criptosporidiose?

( ) Sim

( ) Não 